effect of simvastatin after experimental myocardial infarction (MI).

Methods 60 healthy adult SD rats were randomly divided into the sham operated group, control group, simvastatin group, simvastatin plus L-NAME (inhibitor of NOS) group and simvastatin plus AMG386 (inhibitor of Ang-1) group; Left anterior descending coronary was undergone permanent occlusion to establish the MI model. Rats with MI were administered simvastatin $(1 \mathrm{mg} /(\mathrm{kg} \cdot \mathrm{d}))$, simvastatin plus L-NAME $(40 \mathrm{mg} /(\mathrm{kg} \cdot \mathrm{d}))$, and simvastatin plus AMG386 $(10 \mathrm{mg} /(\mathrm{kg} \cdot \mathrm{wk}))$ respectively for 2 weeks. New microvessels in the ischaemic area near the infarction myocardium were stained by CD31 and the density of new microvessels was dedected; Ang-1, eNOS and phosphoralated endothelial nitric oxide synthase at $\operatorname{Ser}^{1177}$ (p-eNOS) were evaluated by western blotting and RT-PCR assay.

Results (1) simvastatin significantly increased the density of new microvessels $(p<0.05)$, but L-NAME and AMG386 significantly inhibited the pro-angiogenic effect of simvastatin $(p<0.05)$. (2) simvastatin significantly improved The expression of Ang-1, eNOS and $p$-eNOS $(p<0.05)$, and AMG386 significantly decreased simvastatin induced upregulation of $\mathrm{p}-\mathrm{eNOS}$.

Conclusion The pro-angiogenic effect of simvastatin is associated with increased expression of Ang-1, eNOS and p-eNOS, and phosphoralation of eNOS maybe the downstream pathway for Ang-1 induced angiogenesis.

\section{e0045 EFFECTS OF GINSENOSIDE-RBL ON ALDOSTERONE- INDUCED ELASTIN PRODUCTION IN RAT CARDIAC FIBROBLASTS EX VIVO}

doi:10.1136/hrt.2010.208967.45

Hongliang Kong, Zhanquan Li, Weiwei Zhang, Long Yuan, Aijie Hou. Department of Cardiology, Liaoning Provincial People Hospital, Shenyang, China

Objective To investigate whether the role of Ginsenosides-Rbl (Gs-Rb1) on aldosterone-induced elastic fibre deposition of neonatal rat cardiac fibroblasts (CFs) in vitro.

Methods CFs were randomly divided into control group, aldosterone group (10 nmol/l), Gs-Rb1 group (200 umol/l) and Gs-Rb1 binding aldosterone group $(100,200,300,400,500$ umol/1 Gs-Rb1, respectively, basing on $10 \mathrm{nmol} / 1$ aldosterone), all of which were treated for $24 \mathrm{~h}$. MTT colorimetric assay was adopted to evaluate cell proliferation whereas immunofluorescence cytochemistry and western blot were used to detect elastin, tropoelastin synthesis and elastic fibre deposition.

Results 1 . Gs-Rb1 significantly inhibited CFs proliferation induced by aldosterone in a dose-dependent manner $(p<0.01)$. 2. Aldosterone significantly increased elastic fibre deposition, the expression of elastin $(p=0.024)$ and tropoelastin collagen $(p=0.031)$ in CFs. 3. Pretreatment with Gs-Rb1 significantly inhibited the above aldosterone effects, including elastin levels $(p<0.01)$, tropoelastin synthesis $(\mathrm{p}<0.01)$ and elastic fibre deposition in a dose-dependent manner.

Conclusions Gs-Rb1 was shown to inhibit aldosterone-induced collagen production in CFs.

\section{e0046 ROSIGLITAZONE ATTENUATES MYOCARDIAL REMODELLING IN SPONTANEOUSLY HYPERTENSIVE RATS}

\section{doi:10.1136/hrt.2010.208967.46}

Ti Yun, Hao Ming-Xiu, Li Chuan-Bao, Wang Zhi-Hao, Hou Xiao-Yang, Liu Jun-Ni, Zhang Wei, Zhang Yun. Shandong University Qilu Hospital

Background Rosiglitazone, an important Peroxisome proliferatoractivated receptor- $\gamma$ (PPAR- $\gamma$ ) agonist, improves left ventricular hypertrophy in diet-induced hypercholesterolemic rats. However, the effects of rosiglitazone on cardiac remodelling in spontaneous hypertension rats are unclear.

Methods 20 male 8-week-old SHRs were randomly divided into two groups: one treated with oral saline $(n=10)$ and the other treated with rosiglitazone $(5 \mathrm{mg} / \mathrm{kg} / \mathrm{d})(\mathrm{n}=10)$, compared with ten agematched Wistar-Kyoto (WKY) rats as a control group. Echocardiography, immunohistochemistry, real-time RT-PCR, co-immunoprecipitation, and Western blot analysis were performed to assess the effects of rosiglitazone.

Results After 16 weeks of treatment, rosiglitazone decreased left ventricular weight (LVW) to body weight (BW) ratio $(2.35 \pm 0.11 \mathrm{vs}$ $2.56 \pm 0.14 \mathrm{mg} / \mathrm{g}, \mathrm{p}<0.01)$. According to echocardiography, thickening of interventricular septum and posterior wall was prevented $(2.07 \pm 0.03$ vs $2.15 \pm 0.04 \mathrm{~mm}, \mathrm{p}<0.01 ; 2.08 \pm 0.05$ vs $2.15 \pm 0.05 \mathrm{~mm}$, $\mathrm{p}<0.01$, respectively) and midwall fractional shortening (MFS) was improved $(23.82 \pm 0.23 \%$ vs $23.33 \pm 0.4 \%, p<0.01)$ by rosiglitazone. Rosiglitazone decreased collagen I and III mRNA expression (0.06 \pm 0.01 vs $0.18 \pm 0.01, \mathrm{p}<0.01 ; 0.05 \pm 0.01$ vs $0.13 \pm 0.01, \mathrm{p}<0.01$ respectively), and normalised the MMP-9/TIMP-1 ratio (1.16 \pm 0.12 vs $0.78 \pm 0.18, \mathrm{p}<0.01)$. Furthermore, AP-1 activation $(0.51 \pm 0.10$ vs $0.71 \pm 0.09, \mathrm{p}<0.01)$ and NF- $\kappa \mathrm{B}$ expression $(0.33 \pm 0.04$ vs $0.45 \pm 0.08$ $\mathrm{p}<0.01$ ) were suppressed in treated group.

Conclusion These results suggest that treatment with rosiglitazone will improve myocardial remodelling in hypertension. Taken together, PPAR- $\gamma$ agonist rosiglitazone may exert a protective effect on cardiac remodelling in SHRs by decreasing the expression of AP-1 and NF-KB

\section{e0047 ALPHALINONENIC ACID INHIBITS HIGH GLUCOSEMEDIATED ENDOTHELIAL NEUTROPHIL ADHESION BY DECREASING ADHESION MOLECULE EXPRESSION VIA PIЗKAKT PATHWAY}

doi:10.1136/hrt.2010.208967.47

Zhang Wei, Wang Zikuan, He Yong, Xue Yusheng, Zheng Qiangsun. Department of Cardiology, Tangdu Hospital Fourth Military Medical University, Xi'an, P.R. China

Objective Neutrophil-endothelial adhesion is crucial to vascular injury, the major cause of diabetic vascular complications. We studied the mechanism of cardio-protective effect of Alpha-linolenic acid (ALA).

Methods Human umbilical vein endothelial cells (HUVECs) were cultured in $5.5 \mathrm{mmol} / 1$ and $33 \mathrm{mmol} / \mathrm{l}$ for $72 \mathrm{~h}$. ALA with different concentrations was (were) added with defatted bovine serum albumin as a carrier for $18 \mathrm{~h}$ before(del) incubation with high glucose. The effects of ALA on high glucose-induced activation of endothelial cells were then examined.

Results ALA (10 to $100 \mu \mathrm{mol} / \mathrm{l}$ ) decreased the adhesion of human neutrophilic polymorphonuclear leukocytes (PMN) to HUVECs stimulated with high glucose $(33 \mathrm{mmol} / \mathrm{l})$ for $48 \mathrm{~h}$. However, with a higher concentration, ALA $(200 \mu \mathrm{mol} / \mathrm{l})$ exerted an opposite effect. ALA $(50 \mu \mathrm{mol} / \mathrm{l})$ also inhibited intercellular adhesion molecule-1 (ICAM-1) and P-selectin expressions in HUVECs induced by high glucose. ALA enrichment partially prevented the reduction of Akt phosphorylation caused by high glucose. The inhibitory effects of ALA $(50 \mu \mathrm{mol} / \mathrm{l})$ on high glucose-mediated PMN adherence and endothelial adhesion molecule expression were partially abrogated by pretreatment with the PI3K inhibitor LY294002 and wortmannin, suggesting that Akt activation might inhibit activation of endothelial cell induced by high glucose.

Conclusions We conclude that(del) ALA, with a low concentration, acts directly on endothelial cell to inhibit expression of adhesion molecules and neutrophil adhesion mediated by high glucose via a PI3K/Akt-dependent pathway. 